\title{
Review Article \\ Prevalence of Latent Tuberculosis Infection in the Middle East and North Africa: A Systematic Review
}

\author{
Mazin Barry (10 \\ Department of Internal Medicine, Division of Infectious Diseases, College of Medicine, King Saud University, Riyadh, Saudi Arabia \\ Correspondence should be addressed to Mazin Barry; mbarry@ksu.edu.sa
}

Received 21 October 2020; Revised 8 January 2021; Accepted 12 January 2021; Published 29 January 2021

Academic Editor: Stefano Centanni

Copyright ( 2021 Mazin Barry. This is an open access article distributed under the Creative Commons Attribution License, which permits unrestricted use, distribution, and reproduction in any medium, provided the original work is properly cited.

\begin{abstract}
Objective. Data on the prevalence of latent tuberculosis infection (LTBI) in Middle Eastern and North African countries are scarce. We aimed to review all relevant published data in countries belonging to this region to determine the overall prevalence of LTBI in the Middle East and North Africa (MENA) region. Methods. In this systematic review PubMed and Google Scholar databases were searched for observational, prospective, retrospective, cross-sectional, and cohort studies providing prevalence data of LTBI in any MENA country. Studies fulfilling the search criteria were incorporated in the review. Overall prevalence of LTBI with $95 \%$ confidence intervals (CI) was calculated using the random-effects model; heterogeneity was assessed using $I^{2}$ statistics. Gender and age group-based subgroup analyses were performed to evaluate the basis of heterogeneity. Results. The total number of overall LTBI studies identified was 956, of which 31 studies from ten countries within the MENA region were included that represented 12,439 subjects. The overall prevalence was $41.78 \%$ (95\% CI $31.18 \%$ to $52.78 \%, I^{2}=99.31 \%$ ). By gender-based subgroup analysis, the prevalence of LTBI was $33.12 \%$ (95\% CI $18.97 \%$ to $49.04 \%, I^{2}=99.25 \%$ ) and $32.65 \%$ (95\% CI $19.79 \%$ to $47 \%, I^{2}=98.89 \%$ ) in males and females, respectively, while in the age-based subgroup analysis, the prevalence of LTBI was $0.44 \%$ ( $95 \%$ CI $-0.05 \%$ to $0.9 \%$ ), $3.37 \%$ (95\% CI $2.23 \%$ to $4.74 \%, I^{2}=0 \%$ ), and $43.81 \%$ (95\% CI $33.09 \%$ to $54.82 \%, I^{2}=99.18 \%$ ) for children, adolescents, and adults, respectively. Conclusion. This systematic review reveals a high prevalence of LTBI in the MENA region; enhanced LTBI surveillance and prompt infection prevention steps are urgently needed to prevent active tuberculosis, this would help achieve the World Health Organization End TB Strategy 2035, and the United Nations Sustainable Development Goals 2030 target in the MENA region.
\end{abstract}

\section{Introduction}

Tuberculosis (TB) is a major health problem, with an estimated 10 million people (range 9 to 11.1 million) developing TB disease in 2018, of which 5.8 million, 3.2 million, and 1 million were men, women, and children, respectively. Twothirds of cases were from eight countries, India (27\%), China (9\%), Indonesia (8\%), Philippines (6\%), Pakistan (5\%), Nigeria (4\%), Bangladesh (4\%), and South Africa (3\%) [1]. Latent tuberculosis infection (LTBI) does not induce infectious expression of the disease, although it causes continuous immune response generated towards TB antigens. LTBI has a $10 \%$ probability of progressing into active TB disease, $5 \%$ during the first two years of acquiring the infection, and 5\% during the rest of the individual's lifetime. The detection of LTBI and prevention before it becomes infectious is a crucial component of the WHO-End TB strategy. It has been reported from mathematical models that approximately $30 \%$ of the population worldwide are LTBI carriers [2]. Previous studies have documented the rates of LTBI to be $31.2 \%$ in Ethiopia [3], 49\% in Uganda [4], 55.2\% in South Africa [5], 11.2\% in Spain [6], 50\% in India [7], 51\% in Korea [8], and 7.6\% in England [9];however, very few studies have been undertaken to estimate the prevalence of LTBI in the Middle East and North Africa (MENA) region.

In previous studies, it has been observed that patients belonging to lower socioeconomic groups, refugees, and migrants [10], patients with abnormal immune responses 
(post-organ transplant, hemodialysis patients, people living with HIV, etc.), and chronic inflammatory conditions have an increased risk of acquiring TB and its progression to active disease [11-13];further, LTBI in people living with HIV has a $10 \%$ probability of progressing into active $\mathrm{TB}$, when left untreated, annually; furthermore, it has been shown that a significant geographical variation in $\mathrm{TB}$ infection rates persists across the world, implying that health care workers (HCW) in various countries encounter different risks of acquiring TB [14]. In 2018, 87\% of new TB cases occurred in the top thirty high TB burden countries, of which eight countries accounted for two-thirds of all new TB cases, they include India, China, Indonesia, Philippines, Pakistan, Nigeria, Bangladesh, and South Africa, while the occurrence was extremely low in the MENA regions [1], it has also been reported that HCW are at particular risk of LTBI, and hence, annual screening is performed in most standardized health care facilities. In addition, the prevalence of LTBI in HCW has been reported to be higher than that of other community groups around the world $[15,16]$.

Currently, the direct diagnosis of LTBI is not fully possible [17]. The diagnosis of memory $\mathrm{T}$-cell response against LTBI is performed by either the tuberculin skin test (TST) or interferon-gamma release assays (IGRA) [18]. At present, no gold standard test has been developed to measure LTBI; however, there are increasing advancements in this field looking into tumor necrosis factor, chemokines, interleukin growth factors, and other factors that could enhance LTBI diagnosis [19]. With TST, TB-purified protein derivative (PPD) stimulates a type IV hypersensitivity-delayed type reaction [20-22], its advantage is that it is inexpensive and generally accepted especially in low economic countries including Africa [3], but has several disadvantages, as it has demonstrated poor response in individuals with reduced immunity and those with active $\mathrm{TB}$, requires two-step verification, is operative dependent, and exhibits low specificity in determining reactivation of TB in Bacillus Calmette-Guérin (BCG) vaccinated individuals, it can also cause falsepositive results in patients sensitized to naturally existing nontuberculous mycobacteria $[18,23]$.

On the other hand, IGRA has greater specificity compared to TST [17], it involves only one blood test after incubation with Mycobacteria tuberculosis-specific antigens, following which $\mathrm{T}$-cell mediated immune response and interferon- (IFN-) gamma release are measured. The QuantiFERON ${ }^{\circledR}$-TB-Gold-in-Tube (QFT-GIT) and T-SPOT.TB assay tests are the two commercially available IGRA, in which the former is based on ELISA (enzyme-linked immunosorbent assay) and comprises of peptides from the ESAT-6, CFP-10, and TB7.7 antigens of TB. T-SPOT.TB assay is preferred in immunocompromised patients [2426]. IGRA provides more conclusive results that would help in decision-making, with only a single visit required for the test, it also eliminates false-positive results in people vaccinated with BCG or sensitized with nontuberculous mycobacteria.

Several previous studies have documented the prevalence of LTBI in many countries of the Middle East and North Africa, in a wide range of population, including HCW, household contacts, people living with HIV, prisoners, refugees, and in patients with varied health problems; however, to our knowledge, there are no published studies that have assessed the overall prevalence within the whole MENA region; hence, we performed a systematic review to evaluate the prevalence of LTBI in the MENA region in different population groups belonging to various age groups.

\section{Methods}

\subsection{Criteria for Considering Studies}

2.1.1. Inclusion Criteria. Studies based on the incidence or prevalence of LTBI among people of all ages, origin, socioeconomic, and educational backgrounds, in countries located in the Middle East and North Africa, that are cross-sectional, observational, cohort, prospective, and retrospective studies, with LTBI detection performed with either TST or IGRA or both.

2.1.2. Exclusion Criteria. Systematic reviews, case reports, case series, editorials, letters to the editors, and randomized controlled trials.

2.2. Search Strategy. The author searched PubMed and Google Scholar databases for articles published between January 1, 2000 and November 30, 2018, in the English language. The use of medical subject heading (MeSH) terms for LTBI was employed in the database search combined with the following search terms: (latent tuberculosis OR TB OR LTBI OR Mycobacterium tuberculosis) AND (Prevalence OR Epidemiology OR “Country name”). The Middle East countries included were Iran, Iraq, Saudi Arabia, Yemen, Syria, Jordan, United Arab Emirates, Israel, Lebanon, Oman, Kuwait, Qatar, Bahrain, Palestine, Cyprus, and Turkey. North African countries included were Egypt, Libya, Algeria, Morocco, Tunisia, Sudan, Western Sahara, and Mauritania. A broad search strategy was used to ensure that all relevant studies were identified, with no filters included in the searches. Following this, the author independently analyzed the title of the study and its abstract and keywords outlining the record, based on which studies were either included or excluded. No minimal sample size was required to be included in the analysis; however, a sample size of $\geq 200$ was considered as adequate, and a sample size of $<200$ was considered as inadequate.

\subsection{Data Extraction}

2.3.1. Study Selection and Data Extraction. A detailed search of PubMed and Google Scholar databases by employing various search terms was performed. The duplicate citations were removed, and the studies for inclusion in the review were selected. The initial screening was based on the citation titles and abstracts, following which, the articles were selected and picked up and their complete text obtained, reviewed, and assessed for their eligibility for inclusion. The bibliographic information of the included studies was also screened to identify additional relevant articles for inclusion; furthermore, the data from relevant studies were abstracted using 


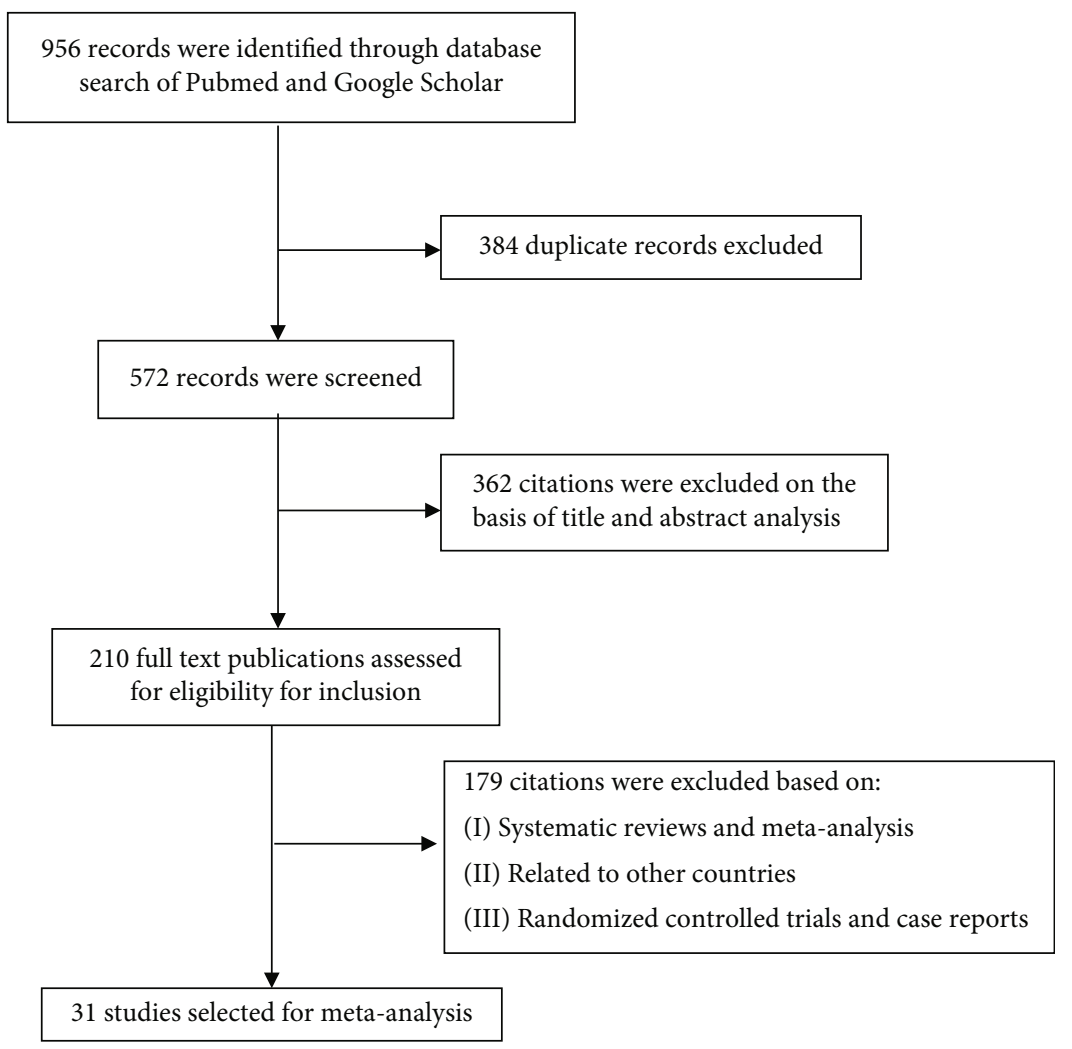

Figure 1: Flowchart for study selection.

a data extraction form, and the applicable items for the review were reported in the PRISMA (Preferred Reporting Items for Systematic Reviews and Meta-Analyses) checklist. The following key information has been presented in the data extraction template: first author, period of study and year of publication, country where the research was conducted, study design, number of participants, age at assessment, tools used for assessment, and key findings.

2.3.2. Quality (Risk of Bias) Assessment. The Mirza and Jenkins [27] checklist were referred to for investigating the quality of included studies. The assessment was based on the following nine criteria: clear study aims, adequate sample size, representative sample, inclusion and exclusion criteria, adequate assessment of outcome, response rate reported, adequate description of data, appropriate statistical analysis, and appropriate informed consent obtained. A final total score was calculated for each of the criteria, scored 0 if absent and 1 if present. Thus, the minimum and maximum obtainable scores would be 0 and 9 , respectively.

2.4. Statistical Analysis. Analysis was performed using STATA software. The effect sizes were reported as proportions with $95 \%$ confidence intervals. The heterogeneity of effects was assessed and quantified by the $I^{2}$. The $I^{2}$ values greater than $50 \%$ were considered to represent substantial heterogeneity. The random-effects model was subjected in cases exhibiting substantial heterogeneity. Subgroup analysis based on sex (male and female), by age strata, and by quality score of the studies $(<5$ and $\geq 5)$ was also performed. A $p$ value less than 0.05 was considered statistically significant for all the analyses undertaken.

\section{Results}

3.1. Search Results and Study Selection. The database search resulted in a total of 956 citations, of which 384 citations were eliminated due to their duplication, and the rest of the 572 citations were examined. After screening, examination of titles and abstracts resulted in the elimination of 362 citations from the study. Following this, 210 full-text citations were retrieved, and after subjecting them to inclusion and exclusion criteria, a total of 31 studies were identified (Figure 1).

3.2. Study Characteristics. Thirty-one studies representing 12,439 subjects from ten countries within the MENA region were included: thirteen from Turkey, five from both Iran and Saudi Arabia, two from Egypt, and one each from Syria, Israel, Oman, Qatar, Tunisia, and United Arab Emirates. These studies were conducted between 2005 till 2018. The sample size ranged from 34 to 2,650 (Table 1).

3.3. Publication Bias. From the 31 studies, the minimal checklist score was 5 in two studies, while the highest was 9. Details of all included studies clarity, adequacy of sample size, and other details are outlined in Table 2.

3.4. Prevalence of LTBI. The prevalence of LTBI was assessed in 31 studies using random-effects model. A total of 3,981 events were observed among the 12,439 subjects. The proportion of LTBI ranged from $0.44 \%$ to $88.15 \%$. The overall 


\begin{tabular}{|c|c|c|c|c|c|c|c|c|c|c|c|}
\hline 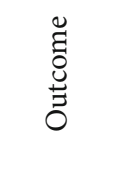 & 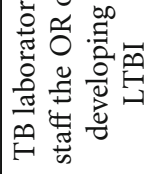 & 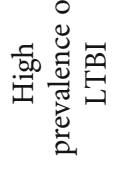 & 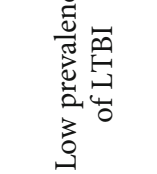 & 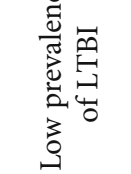 & 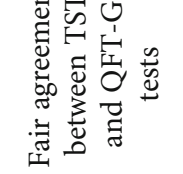 & 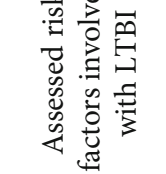 & 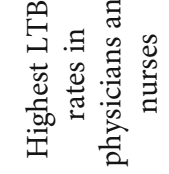 & 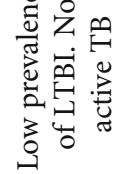 & 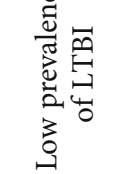 & 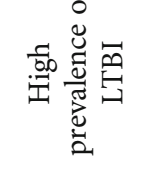 & 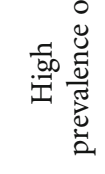 \\
\hline 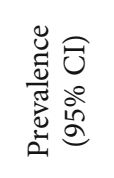 & 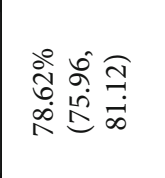 & 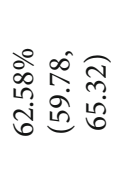 & 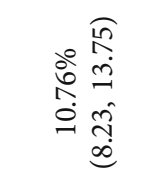 & 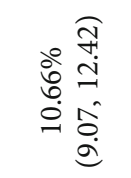 & 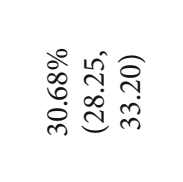 & 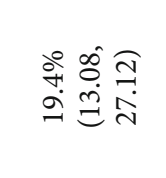 & 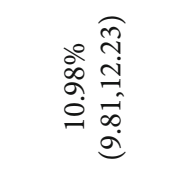 & 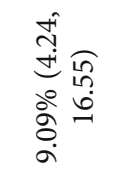 & 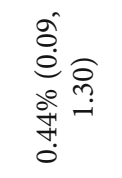 & 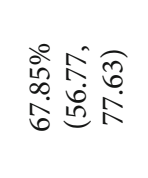 & \\
\hline 兽 & $\overline{\hat{R}}$ & $\stackrel{\circ}{\circ}$ & i & I & $\overrightarrow{\mathcal{F}}$ & $\stackrel{i}{ }$ & $\overrightarrow{\mathrm{N}}$ & $\sigma$ & $m$ & in & $\widetilde{\cong}$ \\
\hline $\begin{array}{l}\frac{n}{0} \\
0 \\
0\end{array}$ & 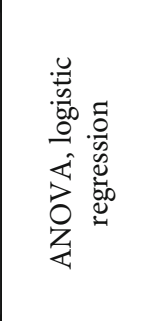 & 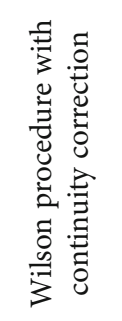 & 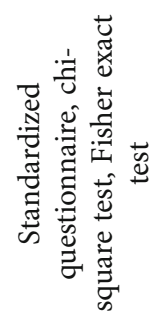 & 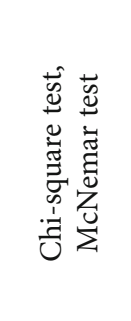 & 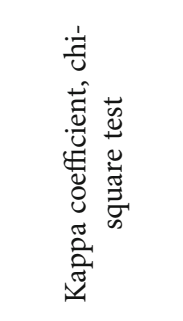 & 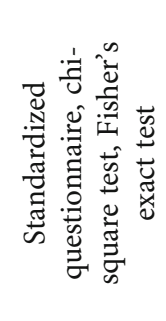 & 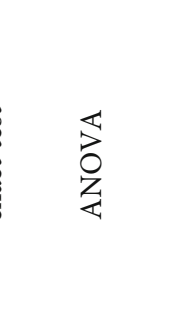 & 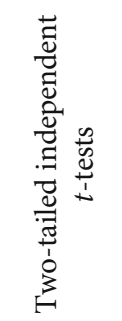 & 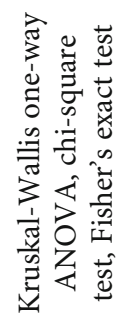 & 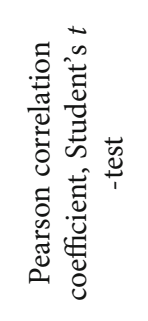 & 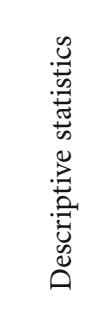 \\
\hline 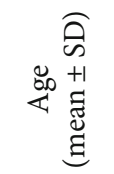 & 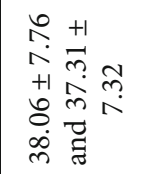 & 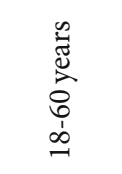 & 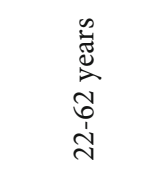 & 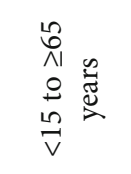 & 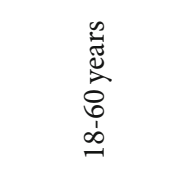 & 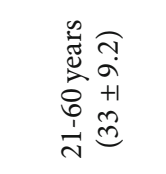 & 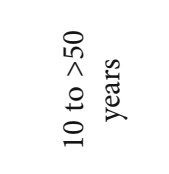 & 战 & 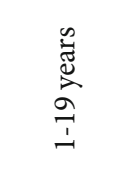 & 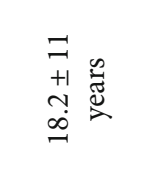 & 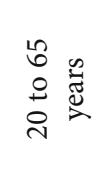 \\
\hline 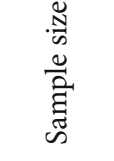 & $\stackrel{\circ}{\circ}$ & $\stackrel{\infty}{\mathbb{\Xi}}$ & స్టి & 욤 & 放 & 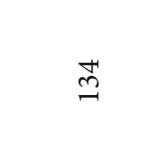 & 呙 & a & 。이 & $\stackrel{\infty}{\infty}$ & $\overrightarrow{\mathrm{N}}$ \\
\hline $\begin{array}{l}\text { 害器 } \\
\text { 题 }\end{array}$ & 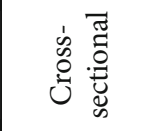 & 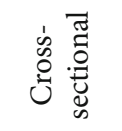 & 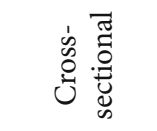 & 它 & 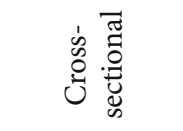 & 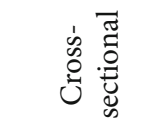 & 它营 & 它 & 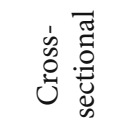 & 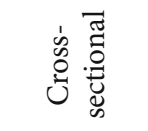 & 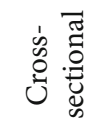 \\
\hline 点苍氙 & 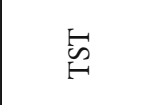 & $\vec{\omega}$ & 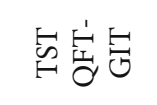 & 㞾宏氙 & 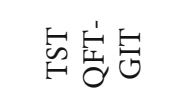 & 岂氙 & $\stackrel{t}{5}$ & 峲氨 & 炭氙 & $\stackrel{\vec{b}}{\vec{H}}$ & \\
\hline 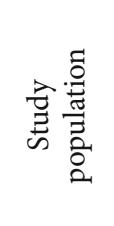 & 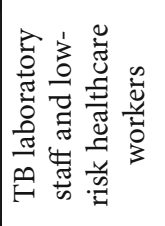 & 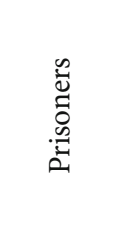 & 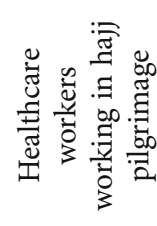 & 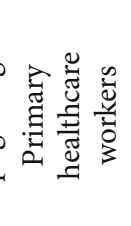 & 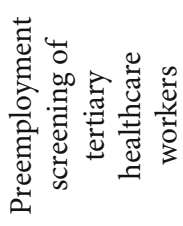 & 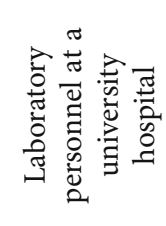 & 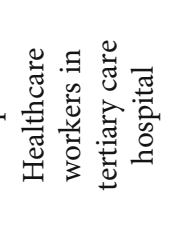 & 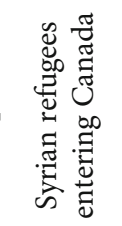 & 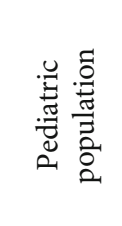 & 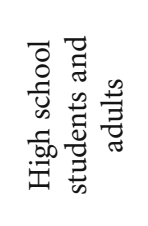 & 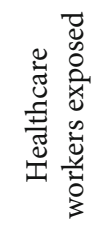 \\
\hline 音 & 岛 & 窇 & 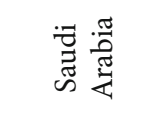 & 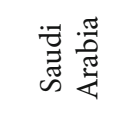 & 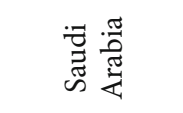 & 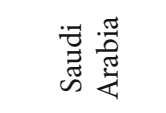 & 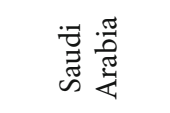 & 莺 & 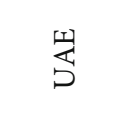 & 离 & 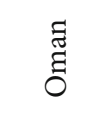 \\
\hline こ्ञ & 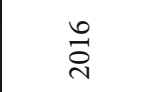 & 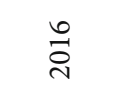 & $\stackrel{\infty}{\stackrel{\text { N }}{*}}$ & $\hat{\vec{i}}$ & $\stackrel{\vec{N}}{\vec{N}}$ & ت্ं & $\stackrel{\circ}{\grave{i}}$ & $\stackrel{\infty}{\stackrel{\sim}{\Delta}}$ & $\stackrel{+}{a}$ & 㝕 & 品 \\
\hline 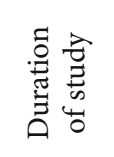 & 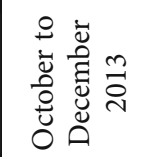 & 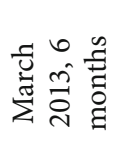 & 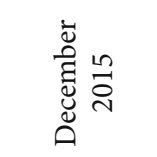 & 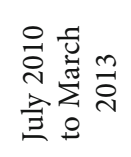 & 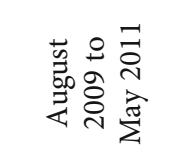 & 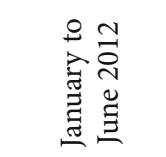 & 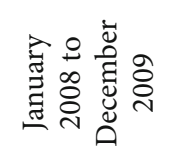 & 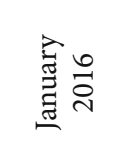 & 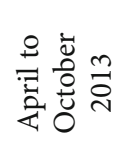 & 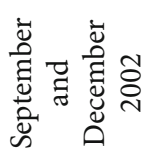 & 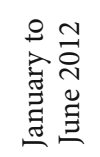 \\
\hline 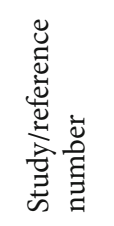 & 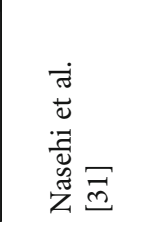 & 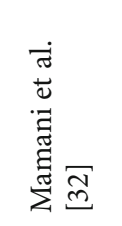 & 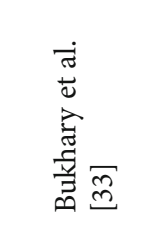 & 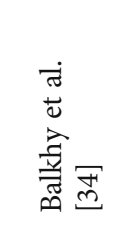 & 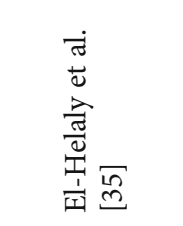 & 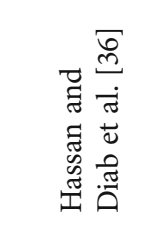 & 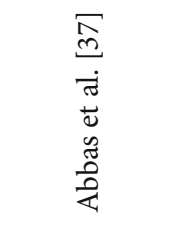 & 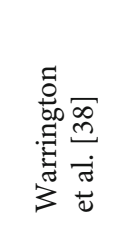 & 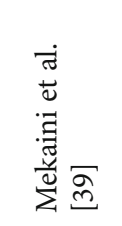 & 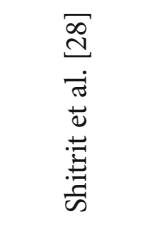 & 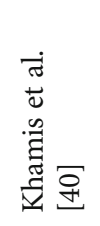 \\
\hline
\end{tabular}




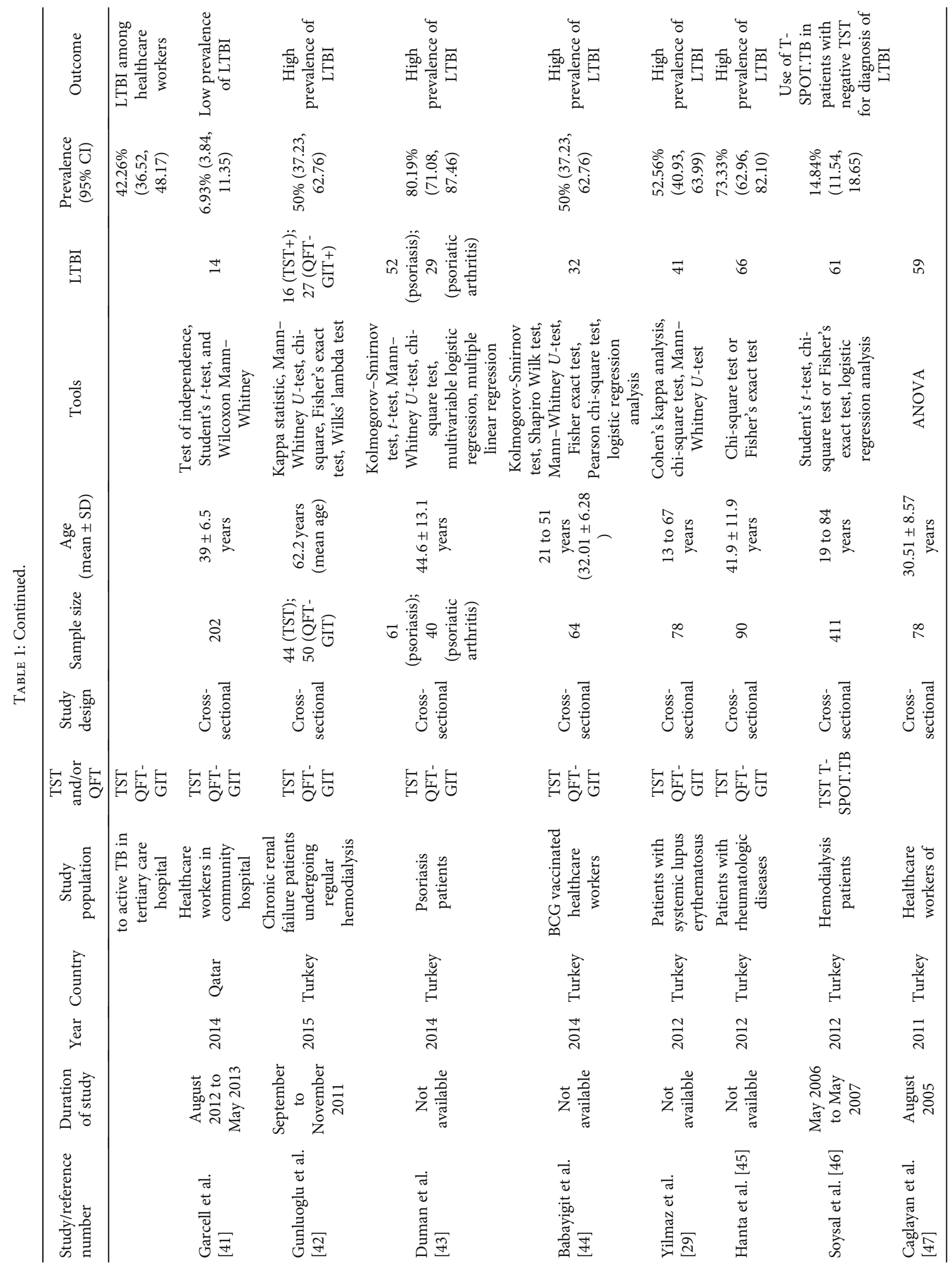




\begin{tabular}{|c|c|c|c|c|c|c|c|c|c|}
\hline 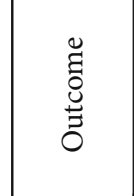 & 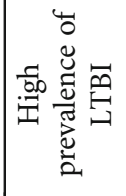 & 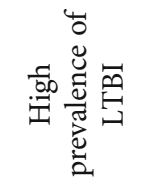 & 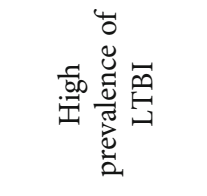 & 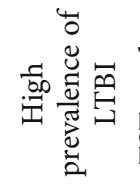 & 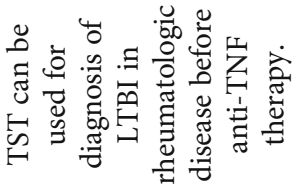 & 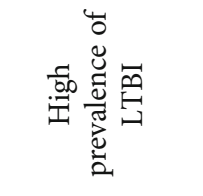 & 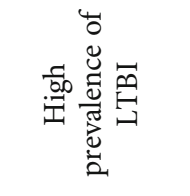 & 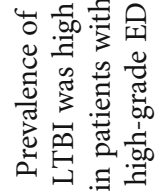 & 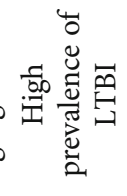 \\
\hline 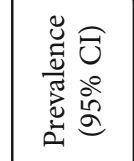 & 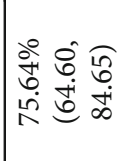 & 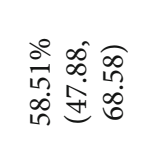 & 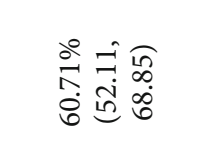 & 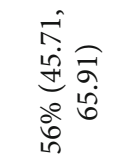 & 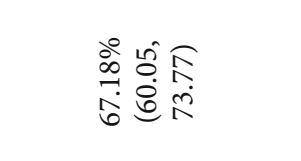 & 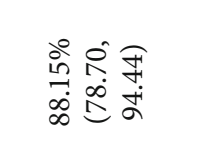 & 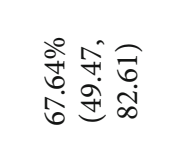 & 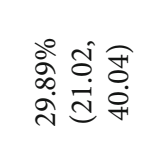 & 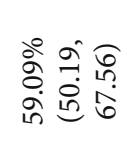 \\
\hline 总 & & in & $\stackrel{\angle \infty}{\infty}$ & 눈 & Әิ & $\hat{\sigma}$ & $\ddot{\sim}$ & શે & $\stackrel{\infty}{\wedge}$ \\
\hline $\begin{array}{l}\frac{n}{0} \\
0 \\
0\end{array}$ & & 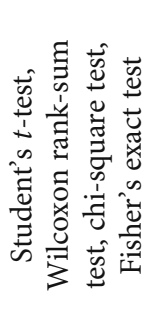 & 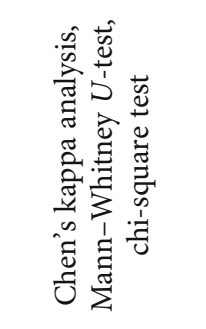 & 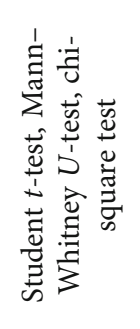 & 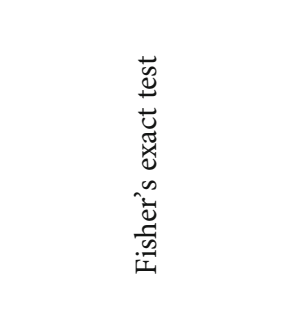 & 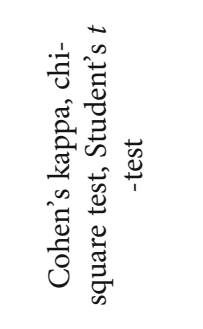 & 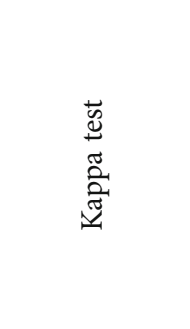 & 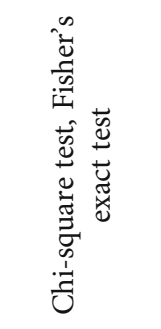 & 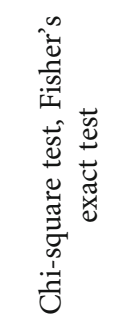 \\
\hline 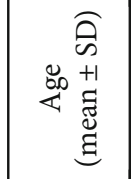 & & 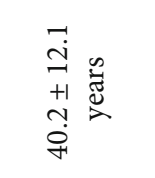 & 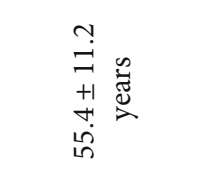 & 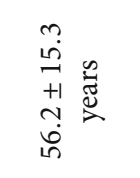 & 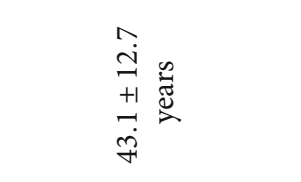 & 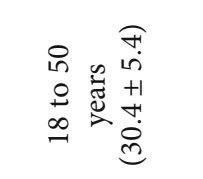 & 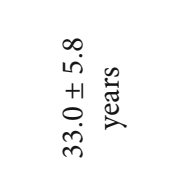 & 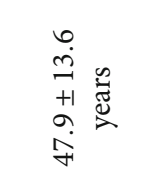 & 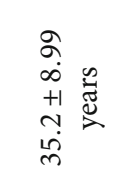 \\
\hline 营 & & హ゙ & g & 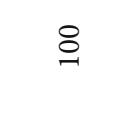 & $\widetilde{\Omega}$ & $\stackrel{\circ}{\curvearrowleft}$ & ఉ' & $\hat{a}$ & $\tilde{\triangleq}$ \\
\hline 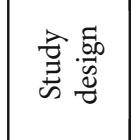 & & 它 & 它 & के & 它 & 它 & 它 & 它 & 它 \\
\hline 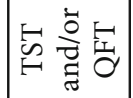 & 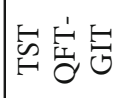 & 氙宏思 & 点崖思 & 点宏曷 & $\vec{b}$ & 点岀曷 & 点崖曷 & 旨宏曷 & 崖宏完 \\
\hline 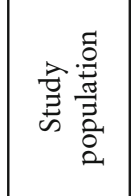 & 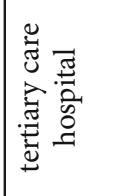 & 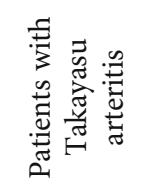 & 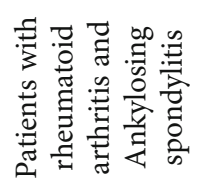 & 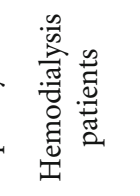 & 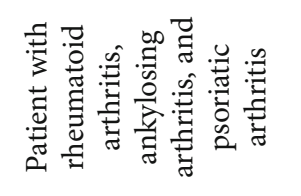 & 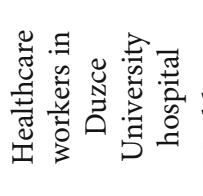 & 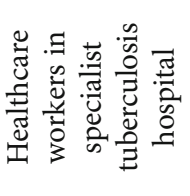 & 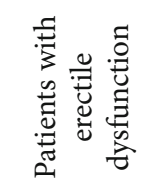 & 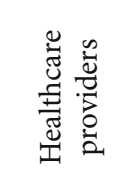 \\
\hline $\begin{array}{l}\text { E् } \\
\text { ठ }\end{array}$ & & 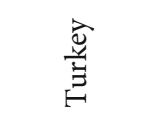 & 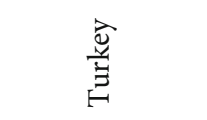 & 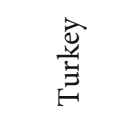 & 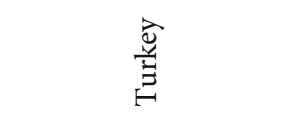 & 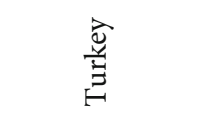 & $\begin{array}{l}\stackrel{\overrightarrow{\bar{v}}}{\grave{\Xi}} \\
\underline{H}\end{array}$ & 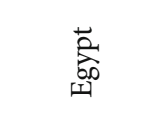 & $\underset{5}{\overrightarrow{0}}$ \\
\hline 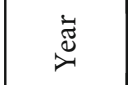 & & $\stackrel{\circ}{\circ}$ & ڤ્̀ે & $\stackrel{\circ}{\stackrel{2}{\sim}}$ & 啇 & ڤ్ & $\underset{\sim}{\stackrel{\imath}{*}}$ & $\stackrel{\infty}{\stackrel{\sim}{n}}$ & 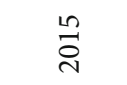 \\
\hline 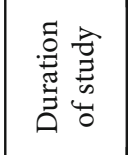 & & 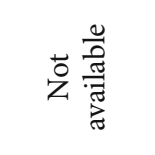 & 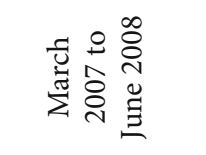 & 萼莺 & 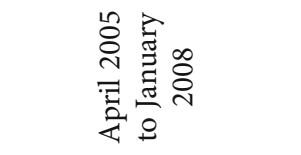 & 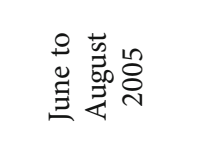 & 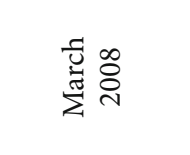 & 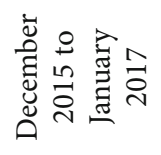 & 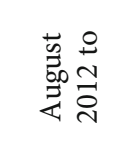 \\
\hline 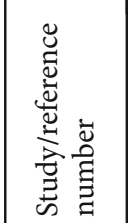 & & 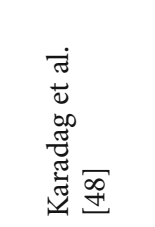 & 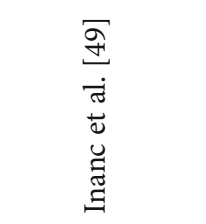 & 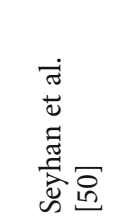 & 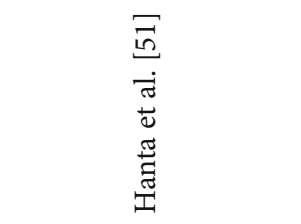 & 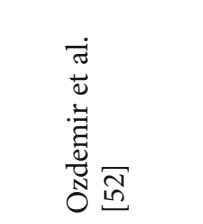 & 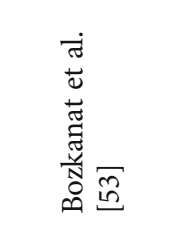 & 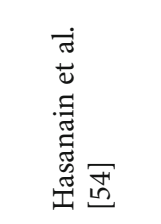 & 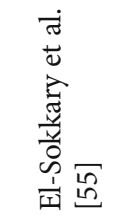 \\
\hline
\end{tabular}




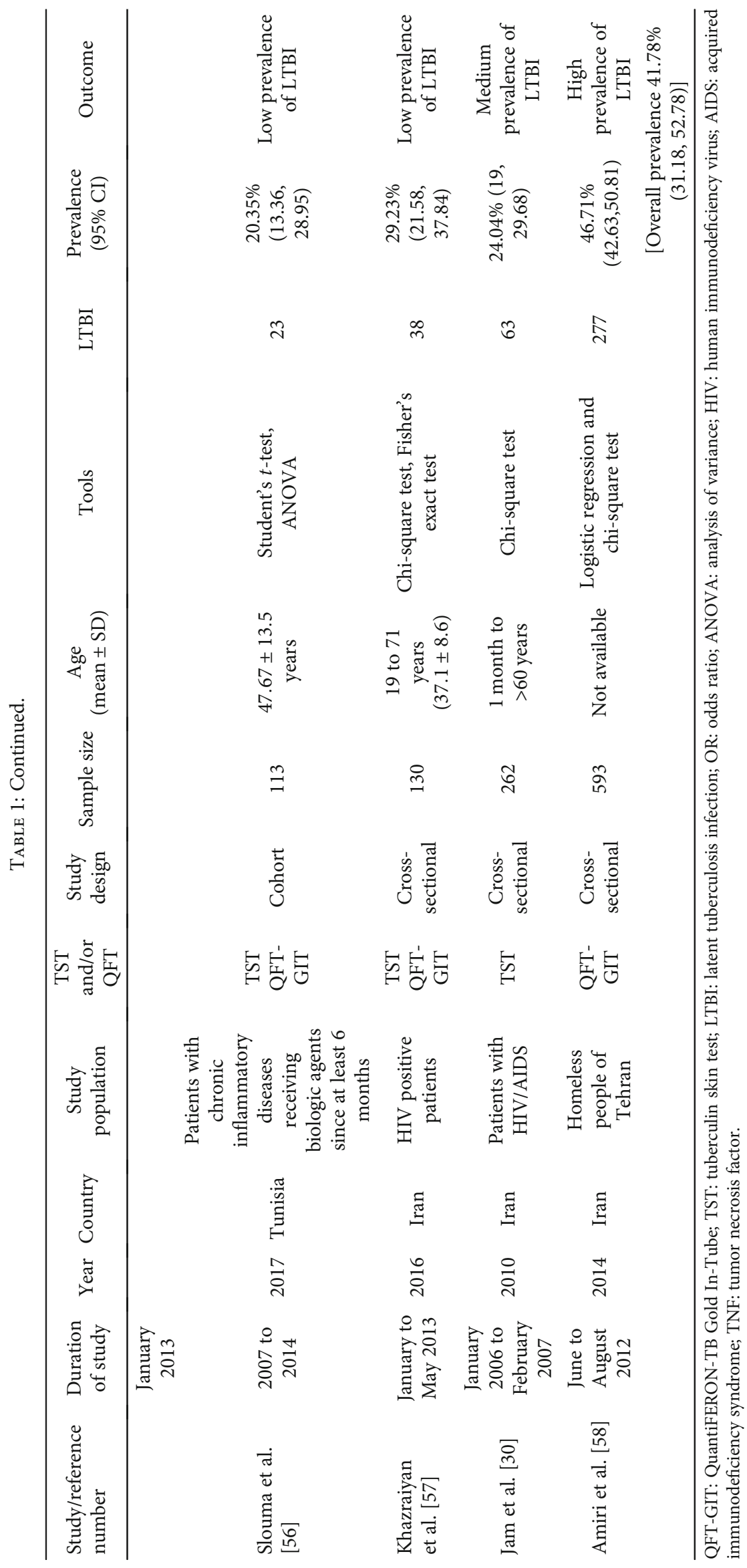


TABLE 2: Quality assessment of the studies included in the review.

\begin{tabular}{|c|c|c|c|c|c|c|c|c|c|c|}
\hline Study & $\begin{array}{l}\text { Clear } \\
\text { study } \\
\text { aims }\end{array}$ & $\begin{array}{l}\text { Adequate } \\
\text { sample } \\
\text { size }\end{array}$ & $\begin{array}{l}\text { Representative } \\
\text { sample }\end{array}$ & $\begin{array}{l}\text { Inclusion } \\
\text { and } \\
\text { exclusion } \\
\text { criteria }\end{array}$ & $\begin{array}{l}\text { Adequate } \\
\text { assessment } \\
\text { of outcome }\end{array}$ & $\begin{array}{l}\text { Response } \\
\text { rate } \\
\text { reported }\end{array}$ & $\begin{array}{c}\text { Adequate } \\
\text { description } \\
\text { of data }\end{array}$ & $\begin{array}{l}\text { Appropriate } \\
\text { statistical } \\
\text { analysis }\end{array}$ & $\begin{array}{l}\text { Appropriate } \\
\text { informed } \\
\text { consent } \\
\text { obtained }\end{array}$ & $\begin{array}{l}\text { Total } \\
\text { score }\end{array}$ \\
\hline $\begin{array}{l}\text { Nasehi et al., } \\
2016 \text { [31] }\end{array}$ & 1 & 1 & 1 & 1 & 1 & 1 & 1 & 1 & 1 & 9 \\
\hline $\begin{array}{l}\text { Mamani } \\
\text { et al., } 2016 \\
\text { [32] }\end{array}$ & 1 & 1 & 1 & 1 & 1 & 1 & 1 & 1 & 1 & 9 \\
\hline $\begin{array}{l}\text { Bukhary } \\
\text { et al., } 2018 \\
{[33]}\end{array}$ & 1 & 1 & 1 & 1 & 1 & 0 & 1 & 1 & 1 & 8 \\
\hline $\begin{array}{l}\text { Balkhy et al., } \\
2017 \text { [34] }\end{array}$ & 1 & 1 & 1 & 1 & 1 & 0 & 1 & 1 & 1 & 8 \\
\hline $\begin{array}{l}\text { El-Helaly } \\
\text { et al., } 2014 \\
{[35]}\end{array}$ & 1 & 1 & 1 & 1 & 1 & 0 & 1 & 1 & 0 & 7 \\
\hline $\begin{array}{l}\text { Hassan and } \\
\text { Diab, } 2014 \\
{[36]}\end{array}$ & 1 & 0 & 1 & 1 & 0 & 0 & 1 & 1 & 1 & 6 \\
\hline $\begin{array}{l}\text { Abbas et al., } \\
2010 \text { [37] }\end{array}$ & 1 & 1 & 1 & 1 & 1 & 0 & 1 & 1 & 0 & 7 \\
\hline $\begin{array}{l}\text { Warrington } \\
\text { et al., } 2018 \\
\text { [38] }\end{array}$ & 1 & 0 & 1 & 1 & 0 & 0 & 1 & 1 & 0 & 5 \\
\hline $\begin{array}{l}\text { Mekaini } \\
\text { et al., } 2014 \\
\text { [39] }\end{array}$ & 1 & 1 & 1 & 1 & 1 & 0 & 1 & 1 & 1 & 8 \\
\hline $\begin{array}{l}\text { Shitrit et al., } \\
2005 \text { [28] }\end{array}$ & 1 & 0 & 1 & 1 & 0 & 1 & 1 & 1 & 1 & 7 \\
\hline $\begin{array}{l}\text { Khamis } \\
\text { et al., } 2016 \\
{[40]}\end{array}$ & 1 & 1 & 1 & 1 & 1 & 0 & 1 & 1 & 0 & 7 \\
\hline $\begin{array}{l}\text { Garcell } \\
\text { et al., } 2014 \\
{[41]}\end{array}$ & 1 & 1 & 1 & 1 & 1 & 0 & 1 & 1 & 0 & 7 \\
\hline $\begin{array}{l}\text { Gunluoglu } \\
\text { et al., } 2015 \\
{[42]}\end{array}$ & 1 & 0 & 1 & 1 & 0 & 1 & 1 & 1 & 1 & 7 \\
\hline $\begin{array}{l}\text { Duman } \\
\text { et al., } 2014 \\
{[43]}\end{array}$ & 1 & 0 & 1 & 1 & 0 & 1 & 1 & 1 & 1 & 7 \\
\hline $\begin{array}{l}\text { Babayigit } \\
\text { et al., } 2014 \\
{[44]}\end{array}$ & 1 & 0 & 1 & 1 & 0 & 1 & 1 & 1 & 1 & 7 \\
\hline $\begin{array}{l}\text { Yilmaz } \\
\text { et al., } 2012 \\
\text { [29] }\end{array}$ & 1 & 0 & 1 & 1 & 0 & 1 & 1 & 1 & 1 & 7 \\
\hline $\begin{array}{l}\text { Hanta et al., } \\
2012 \text { [45] }\end{array}$ & 1 & 0 & 1 & 1 & 0 & 1 & 1 & 1 & 0 & 6 \\
\hline $\begin{array}{l}\text { Soysal et al., } \\
2012 \text { [46] }\end{array}$ & 1 & 0 & 1 & 1 & 0 & 0 & 1 & 1 & 0 & 5 \\
\hline \multirow{2}{*}{$\begin{array}{l}\text { Caglayan } \\
\text { et al., } 2011 \\
{[47]}\end{array}$} & 1 & 0 & 1 & 1 & 0 & 1 & 1 & 1 & 0 & 6 \\
\hline & 1 & 0 & 1 & 1 & 0 & 1 & 1 & 1 & 0 & 6 \\
\hline
\end{tabular}


TABLE 2: Continued.

\begin{tabular}{|c|c|c|c|c|c|c|c|c|c|c|}
\hline Study & $\begin{array}{l}\text { Clear } \\
\text { study } \\
\text { aims }\end{array}$ & $\begin{array}{l}\text { Adequate } \\
\text { sample } \\
\text { size }\end{array}$ & $\begin{array}{l}\text { Representative } \\
\text { sample }\end{array}$ & $\begin{array}{l}\text { Inclusion } \\
\text { and } \\
\text { exclusion } \\
\text { criteria }\end{array}$ & $\begin{array}{c}\text { Adequate } \\
\text { assessment } \\
\text { of outcome }\end{array}$ & $\begin{array}{l}\text { Response } \\
\text { rate } \\
\text { reported }\end{array}$ & $\begin{array}{c}\text { Adequate } \\
\text { description } \\
\text { of data }\end{array}$ & $\begin{array}{c}\text { Appropriate } \\
\text { statistical } \\
\text { analysis }\end{array}$ & $\begin{array}{c}\text { Appropriate } \\
\text { informed } \\
\text { consent } \\
\text { obtained }\end{array}$ & $\begin{array}{l}\text { Total } \\
\text { score }\end{array}$ \\
\hline \multicolumn{11}{|l|}{$\begin{array}{l}\text { Karadag } \\
\text { et al., } 2010 \\
{[48]}\end{array}$} \\
\hline $\begin{array}{l}\text { Inanc et al., } \\
2009 \text { [49] }\end{array}$ & 1 & 1 & 1 & 1 & 1 & 1 & 1 & 1 & 1 & 9 \\
\hline $\begin{array}{l}\text { Seyhan } \\
\text { et al., } 2010 \\
{[50]}\end{array}$ & 1 & 0 & 1 & 1 & 0 & 1 & 1 & 1 & 1 & 7 \\
\hline $\begin{array}{l}\text { Hanta et al., } \\
2008 \text { [51] }\end{array}$ & 1 & 0 & 1 & 1 & 0 & 1 & 1 & 1 & 0 & 6 \\
\hline $\begin{array}{l}\text { Ozdemir } \\
\text { et al., } 2007 \\
{[52]}\end{array}$ & 1 & 0 & 1 & 1 & 0 & 1 & 1 & 1 & 1 & 7 \\
\hline $\begin{array}{l}\text { Bozkanat } \\
\text { et al., } 2016 \\
{[53]}\end{array}$ & 1 & 0 & 1 & 1 & 0 & 1 & 1 & 1 & 0 & 6 \\
\hline $\begin{array}{l}\text { Hasanain } \\
\text { et al., } 2018 \\
{[54]}\end{array}$ & 1 & 0 & 1 & 1 & 0 & 0 & 1 & 1 & 1 & 6 \\
\hline $\begin{array}{l}\text { El-Sokkary } \\
\text { et al., } 2015 \\
\text { [55] }\end{array}$ & 1 & 0 & 1 & 1 & 0 & 1 & 1 & 1 & 1 & 7 \\
\hline $\begin{array}{l}\text { Slouma } \\
\text { et al., } 2017 \\
{[56]}\end{array}$ & 1 & 0 & 1 & 1 & 0 & 0 & 1 & 1 & 0 & 5 \\
\hline $\begin{array}{l}\text { Khazraiyan } \\
\text { et al., } 2016 \\
\text { [57] }\end{array}$ & 1 & 0 & 1 & 1 & 0 & 0 & 1 & 1 & 1 & 6 \\
\hline $\begin{array}{l}\text { Jam et al., } \\
2010[30]\end{array}$ & 1 & 1 & 1 & 1 & 1 & 0 & 1 & 1 & 1 & 8 \\
\hline $\begin{array}{l}\text { Amiri et al., } \\
2014 \text { [58] }\end{array}$ & 1 & 1 & 1 & 1 & 1 & 0 & 1 & 1 & 0 & 7 \\
\hline
\end{tabular}

${ }^{*}$ A sample size of $\geq 200$ was considered as adequate and a sample size of $<200$ was considered as inadequate. ${ }^{\dagger} A$ response rate of $<50 \%$ was considered as low $=0$, and $>50 \%$ was considered as high $=1$.

prevalence was observed to be $41.78 \%$ (95\% CI $31.18 \%$ to $52.78 \%, I^{2}=99.31 \%$ ).

The subgroup analyses revealed the existence of heterogeneity. In the gender-based subgroup analysis, some of the studies failed to mention the gender-based prevalence of LTBI, and hence 14 and 15 studies were excluded from the subgroup analysis of males and females, respectively; hence, the subgroup analysis of males was performed with 17 studies, and that of females with 16 studies. The analysis revealed that the proportion of LTBI ranged from $0.32 \%$ to $86.04 \%$ and from $0.54 \%$ to $90.90 \%$ in males and females, respectively. The overall prevalence was estimated to be $33.12 \%$ (95\% CI $18.97 \%$ to $\left.49.04 \%, I^{2}=99.25 \%\right)$ and $32.65 \%$ (95\% CI $19.79 \%$ to $47 \%, I^{2}$ $=98.89 \%)$ in males and females, respectively.

For the evaluation of age-based prevalence, the WHO classification for age groups was utilized, and the age range for children, adolescents, and adults was taken as $<10$ years, between 10 and 19 years, and >19 years, respectively; further, three studies, Shitrit et al. [28], Yilmaz et al. [29], and Jam et al. [30], were excluded from this subgroup analysis as the age of subjects in those studies overlapped the age range for children, adolescents, and adults, i.e., 12 years and above, 13 to 67 years, and 1 month to above 60 years, respectively. Moreover, there was no differentiation in the age range for the prevalence of LTBI in these studies; hence, the subgroup analysis of children, adolescents, and adults was performed with 1, 2, and 27 studies, respectively. The prevalence of LTBI in children was observed to be $0.44 \%$ (95\% CI $-0.05 \%$ to $0.9 \%)$; the prevalence of LTBI in adolescents and adults ranged from $2.46 \%$ to $3.55 \%$ and $6.93 \%$ to $88.15 \%$, respectively. The overall prevalence was observed to be $3.37 \%$ (95\% CI $2.23 \%$ to $4.74 \%, I^{2}=0 \%$ ) and $43.81 \%$ (95\% CI $33.09 \%$ to $54.82 \%, I^{2}=99.18 \%$ ) for adolescents and adults, respectively. 


\section{Discussion}

After screening 956 studies, a total of 31 scientific papers from ten countries within the MENA region were included in this systematic review [28-58]. The subjects included in these studies were healthcare workers, laboratory staff, medical school students, people living with HIV, and patients with chronic inflammatory diseases. The detection of LTBI in these studies was performed by TST or IGRA or both; furthermore, the studies covered the incidence of LTBI among populations belonging to varying age groups, including children, adolescents, and adults.

In the present study, LTBI prevalence was evaluated by employing the random effects model since high heterogeneity was encountered among studies. The existence of high heterogeneity may have possibly been due to variations in study settings, subjects or participants, methodologies involved, exposure to TB patients, and the control measures taken across the studies.

The overall prevalence of LTBI in the MENA region was found to be $41.78 \%$. In the gender-based subgroup analyses, the prevalence of LTBI was found to be $33.12 \%$ and $32.65 \%$ in males and females, respectively. As for the age-based prevalence, it was assessed to be $0.44 \%, 3.37 \%$, and $43.81 \%$ in children, adolescents, and adults, respectively; therefore, this systematic review implies a high prevalence of LTBI in the MENA region irrespective of gender, and in order to achieve the WHO End TB 2035 objective, there is an immediate need to scale up measures to stop TB disease and enhance LTBI detection within the MENA region.

There are some strengths and limitations within this study that needs to be highlighted; first, as per our findings, this is the first systematic review on the epidemiology and prevalence of LTBI in the MENA region. As for limitations, studies published in English alone have been included, therefore, other reports from countries with high TB incidence that are published in native or other languages other than English, in national or local journals, have not been included; additionally, studies published in journals indexed in PubMed and Google Scholar were included, while other studies may exist that were published in other indexing databases.

To conclude, this review indicates a high prevalence of LTBI in the MENA region despite the high heterogeneity observed. Future studies should aim towards more rigorous assessment of LTBI prevalence within the MENA region to reach exact estimates as the first important step to hamper TB disease diffusion in these countries.

\section{Data Availability}

All data are included in the manuscript.

\section{Conflicts of Interest}

The authors declare that they have no conflicts of interest.

\section{References}

[1] Global tuberculosis report 2018, World Health Organization, Licence: CC BY-NC-SA 3.0 IGO, Geneva, 2018, Jan 2019 (https://www.who.int/tb/publications/global_report/en/).

[2] E. L. Corbett, C. J. Watt, N. Walker et al., "The growing burden of tuberculosis: global trends and interactions with the HIV epidemic," Archives of Internal Medicine, vol. 163, no. 9, pp. 1009-1021, 2003.

[3] M. Legesse, G. Ameni, G. Mamo, G. Medhin, G. Bjune, and F. Abebe, "Community-based cross-sectional survey of latent tuberculosis infection in Afar pastoralists, Ethiopia, using QuantiFERON-TB Gold In-Tube and tuberculin skin test," BMC Infectious Diseases, vol. 11, no. 1, pp. 89-97, 2011.

[4] F. N. Kizza, J. List, A. K. Nkwata et al., "Prevalence of latent tuberculosis infection and associated risk factors in an urban African setting," BMC Infectious Diseases, vol. 15, no. 1, pp. 165-173, 2015.

[5] H. Mahomed, T. Hawkridge, S. Verver et al., "Predictive factors for latent tuberculosis infection among adolescents in a highburden area in South Africa," The International Journal of Tuberculosis and Lung Disease, vol. 15, no. 3, pp. 331-336, 2011.

[6] E. E. Àlvarez-León, E. Espinosa-Vega, E. Santana-Rodríguez et al., "Screening for tuberculosis infection in Spanish healthcare workers comparison of the QuantiFERON-TB Gold InTube test with the tuberculin skin test," Infection Control and Hospital Epidemiology, vol. 30, no. 9, pp. 876-883, 2009.

[7] M. Pai, S. Kalantri, A. N. Aggarwal, D. Menzies, and H. M. Blumberg, "Nosocomial tuberculosis in India," Emerging Infectious Diseases, vol. 12, no. 7, pp. 1311-1318, 2006.

[8] K. Lee, M. K. Han, H. R. Choi et al., "Annual incidence of latent tuberculosis infection among newly employed nurses at a tertiary care university hospital," Infection Control and Hospital Epidemiology, vol. 30, no. 12, pp. 1218-1222, 2009.

[9] P. Khanna, V. Nikolayevsky, F. Warburton, E. Dobson, and F. Drob-niewski, "Rate of latent tuberculosis infection detected by occupational health screening of nurses new to a London teaching hospital," Infection Control and Hospital Epidemiology, vol. 30, no. 6, pp. 581-584, 2009.

[10] M. Mondoni, O. Viganò, M. Ferrarese et al., "Haemoptysis and fever in a young refugee from Somalia," International Journal of Infectious Diseases, vol. 77, pp. 57-60, 2018.

[11] M. Sester, U. Sester, P. Clauer et al., "Tuberculin skin testing underestimates a high prevalence of latent tuberculosis infection in hemodialysis patients," Kidney International, vol. 65, no. 5, pp. 1826-1834, 2004.

[12] C. D. Hamilton, "Infectious complications of treatment with biologic agents," Current Opinion in Rheumatology, vol. 16, no. 4, pp. 393-398, 2004.

[13] American Thoracic Society, "Targeted tuberculin testing and treatment of latent tuberculosis infection," American Journal of Respiratory and Critical Care Medicine, vol. 161, pp. 221247, 2000.

[14] M. J. Field, Tuberculosis in the Workplace, National Academy Press, Washington, DC, 2001.

[15] D. Menzies, M. Pai, and G. Comstock, "Meta-analysis: new tests for the diagnosis of latent tuberculosis infection: areas of uncertainty and recommendations for research," Annals of Internal Medicine, vol. 146, no. 5, pp. 340-354, 2007.

[16] A. Mathew, T. David, K. Thomas et al., "Risk factors for tuberculosis among health care workers in South India: a nested 
case-control study," Journal of Clinical Epidemiology, vol. 66, no. 1, pp. 67-74, 2013.

[17] H. Getahun, A. Matteelli, R. E. Chaisson, and M. Raviglione, "Latent mycobacterium tuberculosis infection," The New England Journal of Medicine, vol. 372, no. 22, pp. 2127-2135, 2015.

[18] J. Keane and B. Bresnihan, “Tuberculosis reactivation during immunosuppressive therapy in rheumatic diseases: diagnostic and therapeutic strategies," Current Opinion in Rheumatology, vol. 20, no. 4, pp. 443-449, 2008.

[19] A. O'Garra, P. S. Redford, F. W. McNab, C. I. Bloom, R. J. Wilkinson, and M. P. R. Berry, "The immune response in tuberculosis," Annual Review of Immunology, vol. 31, no. 1, pp. 475527, 2013.

[20] M. Pai, K. Gokhale, R. Joshi et al., "Mycobacterium tuberculosis infection in health care workers in rural India," JAMA, vol. 293, no. 22, pp. 2746-2755, 2005.

[21] Y. A. Kang, H. W. Lee, H. I. Yoon et al., "Discrepancy between the tuberculin skin test and the whole-blood interferon $\gamma$ assay for the diagnosis of latent tuberculosis infection in an intermediate tuberculosis-burden country," JAMA, vol. 293, no. 22, pp. 2756-2761, 2005.

[22] N. Harada, Y. Nakajima, K. Higuchi, Y. Sekiya, J. Rothel, and T. Mori, "Screening for tuberculosis infection using wholeblood interferon- $\gamma$ and Mantoux testing among Japanese healthcare workers," Infection Control and Hospital Epidemiology, vol. 27, no. 5, pp. 442-448, 2006.

[23] N. N. Chegou, J. Heyckendorf, G. Walzl, C. Lange, and M. Ruhwald, "Beyond the IFN- $\gamma$ horizon: biomarkers for immunodiagnosis of infection with mycobacterium tuberculosis," The European Respiratory Journal, vol. 43, no. 5, pp. 1472-1486, 2014.

[24] D. M. Lewinsohn, M. K. Leonard, P. A. LoBue et al., "Official American thoracic society/infectious diseases society of America/centers for disease control and prevention clinical practice guidelines: diagnosis of tuberculosis in adults and children," Clinical Infectious Diseases, vol. 64, no. 2, pp. 111-115, 2017.

[25] P. Andersen, M. E. Munk, J. M. Pollock, and T. M. Doherty, "Specific immune-based diagnosis of tuberculosis," Lancet, vol. 356, no. 9235, pp. 1099-1104, 2000.

[26] S. M. Arend, K. E. van Meijgaarden, K. de Boer et al., "Tuberculin skin testing and in vitro T cell responses to ESAT- 6 and culture filtrate protein 10 after infection with Mycobacterium marinum or M. kansasii," The Journal of Infectious Diseases, vol. 186, no. 12, pp. 1797-1807, 2002.

[27] I. Mirza and R. Jenkins, "Risk factors, prevalence, and treatment of anxiety and depressive disorders in Pakistan: systematic review," BMJ, vol. 328, no. 7443, p. 794, 2004.

[28] D. Shitrit, G. Izbicki, A. B. Shitrit, M. Raz, J. Sulkes, and M. R. Kramer, "Normal D-dimer levels in patients with latent tuberculosis infection," Blood Coagulation \& Fibrinolysis, vol. 16, no. 1, pp. 85-87, 2005.

[29] N. Yilmaz, S. Zehra Aydin, N. Inanc, S. Karakurt, H. Direskeneli, and S. Yavuz, "Comparison of QuantiFERON-TB Gold test and tuberculin skin test for the identification of latent mycobacterium tuberculosis infection in lupus patients," Lupus, vol. 21, no. 5, pp. 491-495, 2012.

[30] S. Jam, D. Sabzvari, S. SeyedAlinaghi, F. Fattahi, H. Jabbari, and M. Mohraz, "Frequency of mycobacterium tuberculosis infection among Iranian patients with HIV/AIDS by PPD test,” Acta Medica Iranica, vol. 48, no. 1, pp. 67-71, 2010.
[31] M. Nasehi, A. Hashemi-Shahraki, A. Doosti-Irani, S. Sharafi, and E. Mostafavi, "Prevalence of latent tuberculosis infection among tuberculosis laboratory workers in Iran," Epidemiology and Health, vol. 39, p. e2017002, 2017.

[32] M. Mamani, H. Mahmudian, M. M. Majzoobi, and J. Poorolajal, "Prevalence and incidence rates of latent tuberculous infection in a large prison in Iran," The International Journal of Tuberculosis and Lung Disease, vol. 20, no. 8, pp. 1072 1077, 2016.

[33] Z. A. Bukhary, S. M. Amer, M. M. Emara, M. E. Abdalla, and S. A. Ali, "Screening of latent tuberculosis infection among health care workers working in Hajj pilgrimage area in Saudi Arabia, using interferon gamma release assay and tuberculin skin test," Annals of Saudi Medicine, vol. 38, no. 2, pp. 90-96, 2018.

[34] H. H. Balkhy, K. El Beltagy, A. El-Saed et al., "Prevalence of latent mycobacterium tuberculosis infection (LTBI) in Saudi Arabia; population based survey," International Journal of Infectious Diseases, vol. 60, pp. 11-16, 2017.

[35] M. El-Helaly, W. Khan, A. El-Saed, and H. H. Balkhy, "Preemployment screening of latent tuberculosis infection among healthcare workers using tuberculin skin test and QuantiFERON-TB Gold test at a tertiary care hospital in Saudi Arabia," Journal of Infection and Public Health, vol. 7, no. 6, pp. 481-488, 2014.

[36] M. I. Hassan and A. E. Diab, "Detection of latent tuberculosis infection among laboratory personnel at a University Hospital in Eastern Saudi Arabia using an interferon gamma release assay," Journal of Infection and Public Health, vol. 7, no. 4, pp. 289-295, 2014.

[37] M. A. Abbas, N. A. AlHamdan, L. A. Fiala, A. K. AlEnezy, and M. S. AlQahtani, "Prevalence of latent TB among health care workers in four major tertiary care hospitals in Riyadh, Saudi Arabia," The Journal of the Egyptian Public Health Association, vol. 85, no. 1-2, pp. 61-71, 2010.

[38] P. Warrington, G. Tyrrell, K. Choy, L. Eisenbeis, R. Long, and R. Cooper, "Prevalence of latent tuberculosis infection in Syrian refugees to Canada," Canadian Journal of Public Health, vol. 109, no. 1, pp. 8-14, 2018.

[39] L. A. Al Mekaini, O. N. Al Jabri, H. Narchi et al., "The use of an interferon-gamma release assay to screen for pediatric latent tuberculosis infection in the eastern region of the Emirate of Abu Dhabi," International Journal of Infectious Diseases, vol. 23, pp. 4-7, 2014.

[40] F. Khamis, A. Al-Lawati, I. Al-Zakwani et al., "Latent tuberculosis in health care workers exposed to active tuberculosis in a tertiary care hospital in Oman," Oman Medical Journal, vol. 31, no. 4, pp. 298-303, 2016.

[41] H. Guanche Garcell, E. Crespo Ramirez, A. Kindelan Contreras, and G. F. Gutierrez, "Latent tuberculosis infection in healthcare workers at a community hospital in Qatar," Journal of Infection and Public Health, vol. 7, no. 4, pp. 356-359, 2014.

[42] G. Gunluoglu, E. C. Seyhan, R. Kazancioglu et al., "Diagnosing latent tuberculosis in immunocompromised patients measuring blood IP-10 production capacity: an analysis of chronic renal failure patients," Internal Medicine, vol. 54, no. 5, pp. 465-472, 2015.

[43] N. Duman, S. Ersoy-Evans, O. Karadağ et al., "Screening for latent tuberculosis infection in psoriasis and psoriatic arthritis patients in a tuberculosis-endemic country: a comparison of the QuantiFERON ${ }^{\circledR}$-TB Gold In-Tube test and tuberculin skin test," International Journal of Dermatology, vol. 53, no. 10, pp. 1286-1292, 2014. 
[44] C. Babayigit, B. Ozer, C. Ozer, T. Inandi, N. Duran, and O. Gocmen, "Performance of QuantiFERON-TB Gold InTube test and tuberculin skin test for diagnosis of latent tuberculosis infection in BCG vaccinated health care workers," Medical Science Monitor, vol. 20, pp. 521-529, 2014.

[45] I. Hanta, S. Ozbek, S. Kuleci, G. Seydaoglu, and E. Ozyilmaz, "Detection of latent tuberculosis infection in rheumatologic diseases before anti-TNF $\alpha$ therapy: tuberculin skin test versus IFN- $\gamma$ assay," Rheumatology International, vol. 32, no. 11, pp. 3599-3603, 2012.

[46] A. Soysal, D. Toprak, M. Koc, H. Arikan, E. Akoglu, and M. Bakir, "Diagnosing latent tuberculosis infection in haemodialysis patients: T-cell based assay (T-SPOT.TB) or tuberculin skin test?," Nephrol dial transplant, vol. 27, no. 4, pp. 16451650, 2012.

[47] V. Cağlayan, O. Ak, G. Dabak et al., "Comparison of tuberculin skin testing and QuantiFERON-TB Gold-In Tube test in health care workers," Tüberküloz ve Toraks, vol. 59, no. 1, pp. 43-47, 2011.

[48] O. Karadag, K. Aksu, A. Sahin et al., "Assessment of latent tuberculosis infection in Takayasu arteritis with tuberculin skin test and Quantiferon-TB Gold test," Rheumatology International, vol. 30, no. 11, pp. 1483-1487, 2010.

[49] N. Inanc, S. Z. Aydin, S. Karakurt, P. Atagunduz, S. Yavuz, and H. Direskeneli, “Agreement between Quantiferon-TB gold test and tuberculin skin test in the identification of latent tuberculosis infection in patients with rheumatoid arthritis and ankylosing spondylitis," The Journal of Rheumatology, vol. 36, no. 12, pp. 2675-2681, 2009.

[50] E. C. Seyhan, S. Sökücü, S. Altin et al., "Comparison of the QuantiFERON-TB Gold In-Tube test with the tuberculin skin test for detecting latent tuberculosis infection in hemodialysis patients," Transplant Infectious Disease, vol. 12, no. 2, pp. 98-105, 2010.

[51] I. Hanta, S. Ozbek, S. Kuleci, and A. Kocabas, “The evaluation of latent tuberculosis in rheumatologic diseases for anti-TNF therapy: experience with 192 patients," Clinical Rheumatology, vol. 27, no. 9, pp. 1083-1086, 2008.

[52] D. Ozdemir, A. N. Annakkaya, G. Tarhan et al., "Comparison of the tuberculin skin test and the QuantiFERON test for latent mycobacterium tuberculosis infections in health care workers in Turkey," Japanese Journal of Infectious Diseases, vol. 60, no. 2-3, pp. 102-105, 2007.

[53] E. Bozkanat, H. Kaya, O. Sezer et al., "Comparison of tuberculin skin test and QuantiFERON-TB gold in tube test for diagnosis of latent tuberculosis infection in health care workers: a cross sectional study," The Journal of the Pakistan Medical Association, vol. 66, no. 3, pp. 270-274, 2016.

[54] A. F. A. Hasanain, A. M. A. Mahran, A. S. Safwat et al., "Latent tuberculosis infection among patients with erectile dysfunction," International Journal of Impotence Research, vol. 30, no. 1, pp. 36-42, 2018.

[55] R. H. El-Sokkary, A. M. Abu-Taleb, O. S. El-Seifi et al., “Assessing the prevalence of latent tuberculosis among health care providers in Zagazig City, Egypt using tuberculin skin test and QuantiFERON-TB Gold In-Tube Test," Central European Journal of Public Health, vol. 23, no. 4, pp. 324-330, 2015.

[56] M. Slouma, I. Mahmoud, O. Saidane, S. Bouden, and L. Abdelmoula, "Depistage de la tuberculose latente chez les patients candidats a un traitement biologique en Tunisie," Thérapie, vol. 72, no. 5, pp. 573-578, 2017.
[57] H. Khazraiyan, Z. A. Liaei, H. E. Koochak, F. A. Ardalan, Z. Ahmadinejad, and A. Soltani, "Utility of QuantiFERONTB Gold In-Tube test in the diagnosis of latent TB in HIVpositive patients in a medium-TB burden country," Journal of the International Association of Providers of AIDS Care (JIAPAC), vol. 15, no. 2, pp. 101-106, 2015.

[58] F. B. Amiri, M. M. Gouya, M. Saifi et al., "Vulnerability of homeless people in Tehran, Iran, to HIV, tuberculosis and viral hepatitis," PLoS One, vol. 9, no. 6, article e98742, 2014. 